\title{
Krafttraining gleich Krafttraining? Neue Erkenntnisse für die onkologische Trainingstherapie
}

\section{Zusammenfassung}

Bewegungstherapeutische Maßnahmen sind mittlerweile integraler Bestandteil der onkologischen (Früh-)Rehabilitation. Neben dem Ausdauer-und Koordinationstraining ist das Krafttraining ein essenzieller Bestandteil der Bewegungstherapie, um beispielsweise den Nebenwirkungen eines Lymphödems, einer Antiandrogentherapie oder einer Kachexie entgegenzuwirken. Um das Outcome von Bewegungsprogrammen vor dem Hintergrund von verschiedensten Nebenwirkungen von onkologischen Erkrankungen und deren Therapien zu optimieren, sind zwingend weitere Studien notwendig. In der vorliegenden Studie sollte untersucht werden, inwieweit ein intensives Krafttraining einem moderaten Krafttraining hinsichtlich des Kraftzuwachses überlegen ist. Zu diesem Zweck wurden 31 Tumorpatienten ohne Krafttrainingserfahrung (unterschiedlichster Entitäten und Therapiestatus) in eine moderate Trainingsgruppe (MT, n=17) und eine Hypertrophietrainingsgruppe randomisiert $(\mathrm{HT}, \mathrm{n}=14)$. Alle Patienten trainierten zunächst über 8 Wochen 2-mal wöchentlich in einem Kraftausdauerzirkel (je 2 Durchgänge), der 6 Kraftgeräte (je 20 Wiederholungen [Wdh]) für die großen Muskelgruppen beinhaltete. In den folgenden 8 Wochen trainierte die MT weiter bei $20 \mathrm{Wdh}$, wohingegen für die HT der Widerstand so erhöht wurde, dass lediglich 8-12 Wdh möglich waren. Die Maximalkraft der Patienten wurde vor Beginn der Intervention (t0), nach 8 Wochen ( $\mathrm{t} 1$ ) und nach 16 Wochen (t2) mittels eines hypothetischen One-Repition-Maximum- (h1RM-) Tests erfasst. Die Ergebnisse zeigten bei ähnlichem Ausgangsniveau der beiden Gruppen einen signifikant erhöhten Kraftzuwachs für fast alle Muskelgruppen von t1 zu t2 in der HT. Der Mehrwert dieser Ergebnisse ist weniger die Feststellung, dass ein HT hinsichtlich des Kraftzuwachses einem MT überlegen ist, sondern vielmehr, dass ein HT auch für Tumorpatienten gut durchführbar ist. Insofern keine Kontraindikationen bestehen, kann somit intensives Krafttraining v.a. für den Muskelaufbau empfohlen werden. Ob der reine Kraftzuwachs für Nebenwirkungen wie das Fatigue-Syndrom eine zentrale oder eher untergeordnete Rolle spielt, müssen weitere Untersuchungen zeigen.

Schlüsselwörter: Sport, körperliche Aktivität, Krafttraining, Krebs, Supportive Therapie

\section{Einleitung}

Die Ergebnisse großer epidemiologischer Studien zeigen, dass körperliche Aktivität und Sport das Potenzial haben, der Entstehung von Tumorerkrankungen entgegenzuwirken sowie bei bestehenden Tumorerkrankungen das Mortalitätsrisiko zu reduzieren [6, 8, 12]. Darüber hinaus lässt die aktuelle Datenlage darauf schließen, dass gezielte bewegungstherapeutische Maßnahmen Nebenwirkungen von medizinischen Therapien (z.B. Fatigue, Lymphödeme, Harninkontinenz) vermindern und sich letztlich positiv auf die Lebensqualität von Tumorpatienten sowohl während als auch nach der Therapie auswirken [9, 13, 17].

Je nach Indikation spielt ein gezieltes Krafttraining eine zentrale Rolle in der on-
Philip Zimmer, Sergej Borowik, Wilhelm Bloch, Anika Giesen, Lars Gerland, Alexander Schenk, Thomas Elter, Freerk T Baumann

kologischen Bewegungstherapie. Neben dem reinen Kraftzuwachs, der auch bei Patienten unter Therapie beobachtet werden kann, konnten Schmitz et al. [13] zeigen, dass ein intensives Krafttraining sich positiv auf die Symptomatik im Rahmen eines Lymphödems auswirkt. Steindorf et al. [14] beschrieben unlängst weitere positive Einflüsse von Krafttraining sowohl während einer strahlen- als auch während einer chemotherapeutischen Behandlung auf das Fatigue-Syndrom und die Lebensqualität. Letztlich weisen erste Studien darauf hin, dass v.a. kachektische Patienten $[1,10,15]$ sowie Patienten unter Antihormontherapie (z.B. Prostatakrebspatienten) [18] von einer Krafttrainingsintervention profitieren.

Insofern keine Kontraindikationen, wie beispielsweise nicht verheilte Operationsnarben und deutlich erniedrigte Blutwerte, vorliegen, stellt sich generell die Frage, wie ein Krafttraining zu gestalten ist, um positive Effekte zu maximieren. Bislang existieren keine standardisierten Krafttrainingsempfehlungen für Krebspatienten.

Unabhängig von der individuellen Trainingserfahrung, dem Therapiestatus und dem Nebenwirkungsprofil, sollte in der vorliegenden Untersuchung überprüft werden, ob ein klassisches gerätegestütztes Hypertrophietraining (HT) einem moderaten Krafttraining (MT) hinsichtlich des Kraftzuwachses bei Krebspatienten überlegen ist. Da eine Überlegenheit des HT bei Gesunden als gesichert gilt [5], stehen v.a. die Anwendbarkeit und Unbedenklichkeit einer solchen Intervention im Mittelpunkt dieser Studie. 


\section{Methodik}

Im Rahmen des Projekts „onkologische Trainingstherapie“ (OTT) des Centrums für integrierte Onkologie Köln/Bonn und der Deutschen Sporthochschule Köln wurden 31 Patienten mit der Diagnose Krebs für die Studie rekrutiert. Zusätzlich zu dem vor Beginn der Studie eingeholtem positivem Ethikvotum mussten alle Teilnehmer vor Studienbeginn eine medizinische Unbedenklichkeitsbescheinigung vorlegen und eine Einverständniserklärung über die Teilnahme an der Studie unterschreiben.

Eingeschlossen wurden alle Tumorpatienten (während der medizinischen Therapie und in der Nachsorge), die innerhalb einer 8-wöchigen Rekrutierungsphase ihr Training aufnahmen und über keine Krafttrainingserfahrung verfügten (Abb. 1). Ausgeschlossen wurden Patienten mit folgenden Kontraindikationen: starke Blutungsneigung, Thrombozytenwerte unter $10.000 / \mu l$, starke Schmerzen und eine Verstärkung der Schmerzen durch Bewegung, potenziell übertragbare Infektionen (zum Schutz anderer Patienten), Hämoglobinwerte unter 8g/dl Blut in Kombination mit Schwindel. Als weitere Ausschlusskriterien wurden orthopädische, kardiologische und psychische Kontraindikationen berücksichtigt.

Das Krafttraining wurde über einen Zeitraum von 16 Wochen bei 2 Trainingseinheiten pro Woche in einem Kraftausdauer-Gerätezirkel der Firma Milon ${ }^{\circledR}$ (Emersacker, Deutschland), bestehend aus Beinstrecker, Brustpresse, Rudergerät, Beinbeuger, Bauchtrainer, Rückenstrecker sowie Fahrradergometern und Crosswalkern, durchgeführt. Alle Patienten wurden vor Beginn der Studie in eine moderate Trainingsgruppe (MT; n=17) und eine Hypertrophietrainingsgruppe (HT; n=14) randomisiert. Vor der ersten Trainingseinheit (t0) wurde die Maximalkraft der Patienten an allen Geräten mittels eines hypothetischen One-Repition-Maximum- (h1RM-) Tests ermittelt. Dazu wurde vom Testleiter am jeweiligen Gerät ein Widerstand eingestellt, der vom Probanden maximal 20mal in kompletter Ausführung bewegt werden konnte. Anhand eines validierten
Tabellenwerkes konnte, ausgehend von der Höhe des Widerstandes und der erreichten Wiederholungszahl, auf die hypothetische Maximalkraft geschlossen werden $[4,7]$.

Im Sinne einer Gerätegewöhnung führten beide Gruppen zunächst für 8 Wochen ein moderates Krafttraining durch (2 Zirkeldurchgänge, 4 Minuten Warm-up auf Ausdauergerät, 3 Krafttrainingsgeräte mit je 20 Wiederholungen, 4 Minuten Ausdauergerät, 3 weitere Krafttrainingsgeräte). Nach 8 Wochen wurde eine Zwischentestung durchgeführt ( $t 1$ ) und die Belastung für die MT so angepasst, dass weiterhin maximal 20 Wiederholungen an den Krafttrainingsgeräten möglich waren. Die Belastung der HT wurde so eingestellt, dass die Patienten mindestens 8 und maximal 12 Wiederholungen pro Gerät und Durchgang durchführen konnten. In beiden Gruppen wurden über den gesamten Interventionszeitraum die Widerstände im Sinne eines progressiven Trainings so angepasst, dass die vorgegebene maximale Wiederholungszahl nicht überschritten werden konnte. Nach weiteren 8 Wochen wurde eine Abschlusstestung (t2) durchgeführt.

Die statistische Analyse erfolgte mit der Software SPSS Statistics (Version 22) von $\mathrm{IBM}^{\circledR}$. Die Baselinewerte (t0) wurden zunächst mittels eines T-Tests für ungepaarte Stichproben verglichen.

Zur Analyse der h1RM-Werte wurde eine einfaktorielle Varianzanalyse (ANOVA) mit Messwiederholung durchgeführt. Bei signifikanten Unterschieden für die Faktoren Zeit und Gruppe $\times$ Zeit wurde ein Bonferroni-Post-Hoc-Test durchgeführt. Insofern keine Sphärizität gegeben war (Mauchly-Test), wurde eine GreenhouseGeisser-Korrektur vorgenommen. Das Signifikanzniveau betrug für alle Testverfahren $\alpha=0,05$.

\section{Ergebnisse}

Die Adhärenz der Teilnehmer lag über den gesamten Zeitraum bei $100 \%$. Die anthropometrischen Daten, Patientencharakteristika sowie die Baselinewerte ( $\mathrm{t} 0$ ) beider Gruppen sind in Tab. 1 dargestellt.

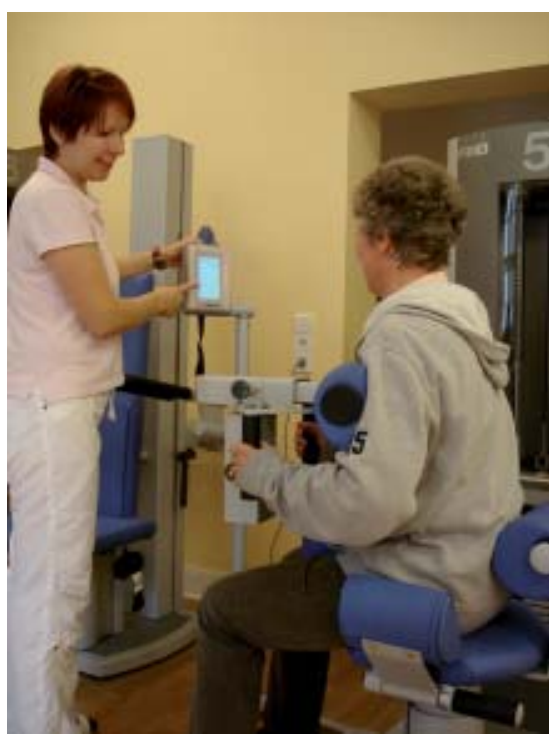

Abb. 1: In die Studie wurden Patienten aufgenommen, die über keine Erfahrung im gerätegestützten Krafttraining verfügten (nachgestellte Situation). Foto: Thieme Verlagsgruppe

Für die Kraftwerte lässt sich mit Ausnahme des Bauchtrainers für alle Geräte bzw. Muskelgruppen eine vergleichbare Entwicklung feststellen. Von t0 zu t1 kam es in keiner der beiden Gruppen zu einem signifikanten Kraftanstieg (Zeit, Gruppe $\times$ Zeit). Die Post-Hoc-Analysen zeigen, dass der Kraftanstieg über die Zeit auf einem Kraftzuwachs in der HT basiert, was letztlich auch in einem signifikanten Unterschied für den Faktor Gruppe resultiert. Von t1 zu t2 kam es in der HT-Gruppe zu einem signifikanten Kraftanstieg, wohingegen dieser Effekt in der MT-Gruppe nicht beobachtet werden konnte. Dementsprechend zeigten sich in der HT-Gruppe auch signifikante Unterschiede der Kraftwerte zwischen to und t2, die in der MTGruppe nicht messbar waren. Die Ergebnisse der ANOVA sind in Tab. 2 und die Verläufe der Kraftwerte aller Trainingsgeräte sind für beide Gruppen in Abb. 2 dargestellt.

\section{Diskussion}

Die Ergebnisse der vorliegenden Studie lassen darauf schließen, dass ein intensives Hypertrophietraining unabhängig von Therapiestatus und Tumorentität gut umsetzbar ist und genau wie bei Nichtbetroffenen zu einem deutlich erhöhten Kraftzu- 
Tab. 1 Patientencharakteristika und Baselinewerte.

\begin{tabular}{|c|c|c|c|}
\hline Parameter & HT & MT & p-Wert \\
\hline$n$ & 14 & 17 & - \\
\hline Geschlecht & $w=6, m=8$ & $w=12, m=5$ & - \\
\hline Nachsorge & 11 & 11 & - \\
\hline Chemotherapie & 0 & 3 & - \\
\hline Antihormontherapie & 3 & 2 & - \\
\hline Strahlentherapie & 0 & 1 & - \\
\hline Entitäten & $\begin{array}{l}\text { Mamma-CA: } 5 \\
\text { Prostata-CA: } 4 \\
\text { NHL: } 1 \\
\text { Ovarial-CA: } 1 \\
\text { Ösophagus-CA: } 1 \\
\text { Kolon-CA: } 1 \\
\text { Rektum-CA: } 1\end{array}$ & $\begin{array}{l}\text { Mamma-CA: } 8 \\
\text { Prostata-CA: } 3 \\
\text { NHL: } 2 \\
\text { Ovarial-CA: } 3 \\
\text { Nierenbecken-CA:1 }\end{array}$ & - \\
\hline Alter & $63(7)$ & $63(11)$ & 0.60 \\
\hline BMI & $25,69(3,57)$ & $24,97(3,4)$ & 0.56 \\
\hline Beinstrecker & $24,8(9,2)$ & $18,9(7,4)$ & 0.08 \\
\hline Beinbeuger & $25,3(11,2)$ & $24,6(14,9)$ & 0,71 \\
\hline Brustpresse & $25,2(16,3)$ & $22,1(10,6)$ & 0,44 \\
\hline Ruderzug & $27,5(19,1)$ & $21,8(10,7)$ & 0,09 \\
\hline Bauchtrainer & $20,9(11,6)$ & $20,8(8,7)$ & 0,82 \\
\hline Rückentrainer & $31,2(16,6)$ & $22,1(9,3)$ & 0,43 \\
\hline
\end{tabular}

Mittelwerte und Standardabweichung für Alter (in Jahren), BMl und h1RM-Werte aller Trainingsgeräte (in kg).

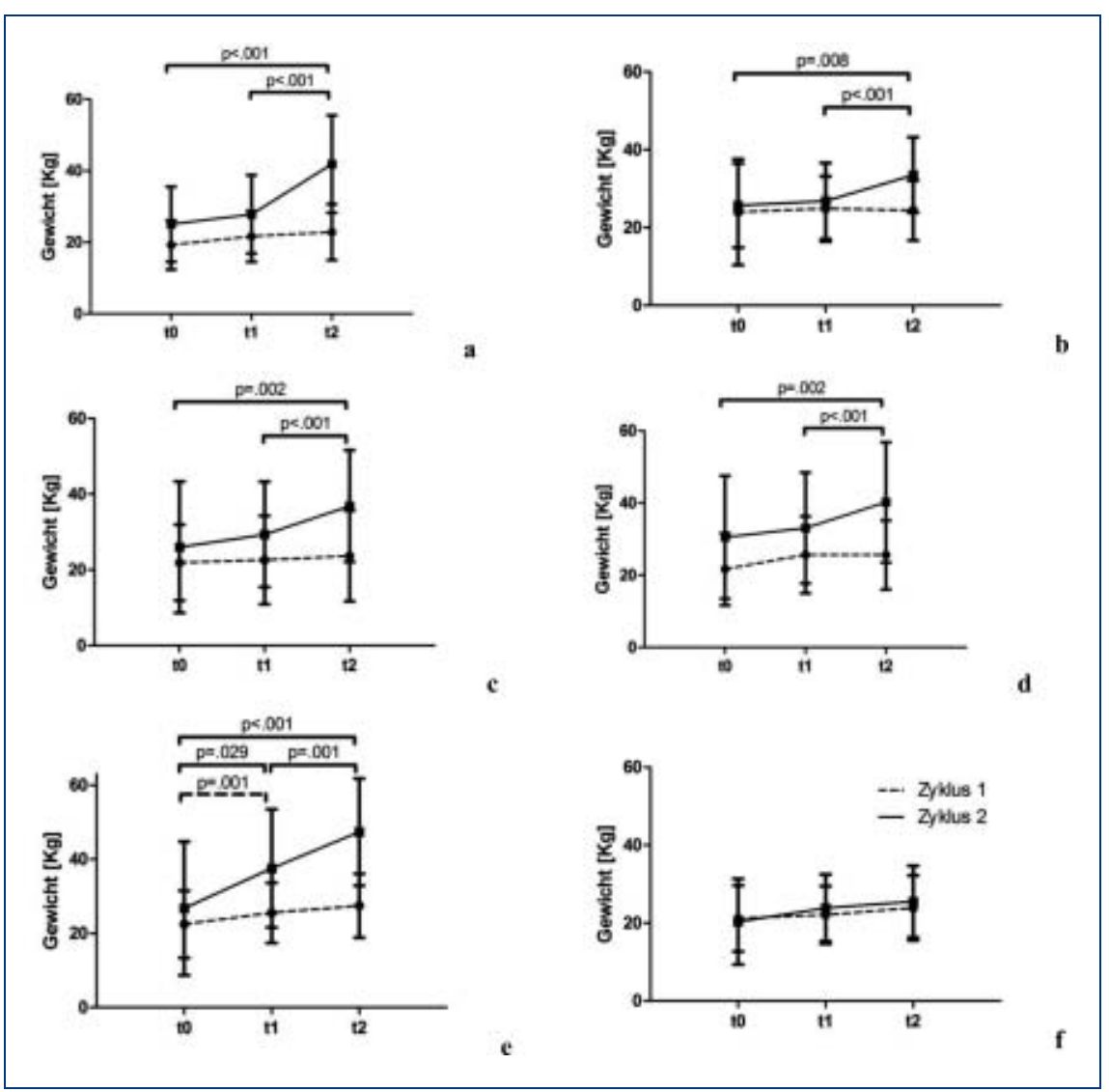

Abb. 2: Zeitliche Verläufe der Kraftwerte für beide Gruppen. a) Beinstrecker, b) Beinbeuger, c) Brustpresse, d) Ruderzug, e) Rückentrainer, f) Bauchtrainer. Signifikante Unterschiede der PostHoc-Tests sind für beide Gruppen separat (Gruppe x Zeit) dargestellt. wachs im Vergleich zu einem moderaten Krafttraining führt [5].

Es stellt sich die Frage, ob im Rahmen eines therapeutischen Krafttrainings der reine Kraftzuwachs für den Patienten der entscheidende Parameter ist, oder ob vielmehr die Reduktion einer bestimmten Nebenwirkung im Fokus stehen sollte. Diese Frage muss letztlich im Kontext der „behandelten“ Nebenwirkung beantwortet werden. Während für kachektische Patienten der Kraft- und damit in Verbindung stehende Muskelmassenzuwachs bzw. -erhalt höchste Priorität hat, könnte es sein, dass eben dieser für einen Patienten mit einem Fatigue-Syndrom eine eher untergeordnete Rolle spielt.

Generell kann aber davon ausgegangen werden, dass sich eine Steigerung der Maximalkraft, besonders bei geschwächten und älteren Patienten, positiv auf deren Alltag auswirkt. Eine gesteigerte Funktionalität, z.B. beim Tragen von Einkäufen und dem Treppensteigen sowie eine verbesserte Sturzprophylaxe bedeuten nicht nur mehr Sicherheit, sondern verbessern auch die soziale Teilhabe und somit insgesamt die Lebensqualität der Patienten.

Letztlich wird zukünftig nicht mehr von Interesse sein, ob ein Tumorpatient trainieren soll, sondern vielmehr wie (Kraft, Ausdauer, Koordination) und mit welcher Intensität ein Bewegungsprogramm vor dem Hintergrund bestimmter Nebenwirkungen zu gestalten ist. Diesbezüglich sind ähnlich wie in der pharmazeutischen Industrie klassische Überlegenheitsstudien notwendig. An dieser Stelle sei angemerkt, dass die häufig v.a. von Ärzten ausgesprochene Maxime „Hauptsache Bewegung“" natürlich nicht falsch ist. Um Patienten während und nach der Therapie im Sinne einer Supportivmaßnahme optimal zu unterstützen, sind jedoch dezidiertere Bewegungsempfehlungen zwingend notwendig.

Die hier vorgestellten Daten müssen im Kontext ihrer Limitationen betrachtet werden. Neben der kleinen Stichprobe schränkt die Heterogenität des untersuchten Patientenkollektivs (Tumorentität, Behandlungsstadium) die Aussagekraft der Ergebnisse ein. Ferner könnte der vergleichsweise starke Kraftzuwachs zumin- 
Tab. 2 Ergebnisse der ANOVA.

\begin{tabular}{|c|c|c|c|c|c|c|c|c|c|}
\hline & HT & & & MT & & & & & \\
\hline & t0 & $\mathrm{t} 1$ & t2 & t0 & t1 & t2 & Zeit & Gruppe & Gruppe x Zeit \\
\hline Beinstrecker & $24,8(3,1)$ & $25,5(3,8)$ & $40,5(5,3)$ & $18,9(1,9)$ & $21,7(2,1)$ & $\begin{array}{l}23 \\
(2,3)\end{array}$ & $\begin{array}{l}\mathrm{df}=1,45 \\
\mathrm{~F}=41,4 \\
\mathrm{p}<, 001\end{array}$ & $\mathrm{p}=, 012$ & $\begin{array}{l}d f=1,45 \\
F=21,2 \\
p<, 001\end{array}$ \\
\hline Beinbeuger & $25,3(3,7)$ & $\begin{array}{l}25 \\
(3,5)\end{array}$ & $32,7(3,7)$ & $24,6(3,9)$ & $24,5(2,5)$ & $24,9(2,2)$ & $\begin{array}{l}d f=1,66 \\
F=5,3 \\
p=, 012\end{array}$ & $\mathrm{p}=, 277$ & $\begin{array}{l}d f=1,66 \\
F=5,15 \\
p=, 014\end{array}$ \\
\hline Brustpresse & $25,2(5,4)$ & $25,3(4,7)$ & $34,9(5,3)$ & $22,1(2,8)$ & $22,5(3,4)$ & $23,7(3,38)$ & $\begin{array}{l}d f=2 \\
F=10,55 \\
p<, 001\end{array}$ & $p=, 166$ & $\begin{array}{l}d f=2 \\
F=5,61 \\
p=0,007\end{array}$ \\
\hline Ruderzug & $27,5(6,4)$ & $29,4(5,7)$ & $39,3(6,4)$ & $21,8(2,8)$ & $\begin{array}{l}25,7 \\
(3)\end{array}$ & $25,7(2,7)$ & $\begin{array}{l}d f=1,45 \\
F=20,65 \\
p<, 001\end{array}$ & $\mathrm{p}=, 047$ & $\begin{array}{l}d f=1,45 \\
F=6,74 \\
p=0,007\end{array}$ \\
\hline Bauchtrainer & $20,9(3,9)$ & $\begin{array}{l}21 \\
(2,5)\end{array}$ & $22,5(2,7)$ & $20,8(2,3)$ & $22,1(2,1)$ & $24,3(2,2)$ & $\begin{array}{l}d f=1,17 \\
F=5,54 \\
p=, 021\end{array}$ & $\mathrm{p}=, 718$ & $\begin{array}{l}d f=1,17 \\
F=0,09 \\
p=, 81\end{array}$ \\
\hline Rückentrainer & $31,2(5,5)$ & $34,7(5,9)$ & $46,6(5,2)$ & $22,1(2,5)$ & $25,4(2,3)$ & $27,8(2,4)$ & $\begin{array}{l}d f=2 \\
F=20,96 \\
p<, 001\end{array}$ & $\mathrm{p}=, 026$ & $\begin{array}{l}d f=2 \\
F=6,7 \\
p=, 003\end{array}$ \\
\hline
\end{tabular}

Für alle Trainingsgeräte sind Mittelwerte und Standardfehler der h1RM-Werte in kg angegeben. Für Faktoren Zeit und Gruppe x Zeit sind jeweils Freiheitsgrade (df), F- und p-Werte dargestellt. Für den Faktor Gruppe sind die Ergebnisse des paarweisen Vergleichs als p-Werte präsentiert.

dest partiell über eine Gewöhnung an das Bewegen größerer Lasten in der Hypertrophiegruppe zu erklären sein. Inwieweit die Geschlechterverteilung (mehr Männer in der HT) einen Einfluss auf den Kraftzuwachs haben könnte, müssen weitere Studien zeigen. Eine Krafttestung an einem Isokineten wäre als Assessment empfehlenswert [11]. Trotzdem spiegeln sowohl die Heterogenität der Patienten als auch die Auswahl des Kraftmessverfahrens ein klassisches, therapeutisches Szenario wieder, was eine Übertragung der Ergebnisse in die Praxis erleichtert.

Basierend auf den Erkenntnissen der hier präsentierten Studie kann ein intensives Krafttraining, ärztliche Unbedenklichkeit vorausgesetzt (Ruhe- und BelastungsEKG sowie Herzsonographie), während der akuten medizinischen Therapie und in der Nachsorge empfohlen werden. An dieser Stelle wird die hohe Relevanz für ein kooperatives Zusammenwirken verschiedener Berufsgruppen (Ärzte, Sportwissenschaftler, Bewegungstherapeuten, etc.) im interdisziplinären Kontext deutlich.

Darüber hinaus ist das Training an modernen Chipkarten-gestützten medizinischen Trainingsgeräten, bei denen Bewegungsamplituden und Gewichte sehr individuell angepasst und kontrolliert werden können, von großem Vorteil. Auf diese
Weise kann nicht nur ein sicheres, optimales und reliables Training gewährleistet, sondern so auch die bewegungstherapeutische Behandlung (z.B. in einer Rehabilitationsklinik), im Sinne einer versorgungsspezifischen Flächendeckung, nahtlos am Wohnort fortgesetzt werden. Weitere Studien sind jedoch zukünftig nötig, um das Outcome bewegungstherapeutischer Interventionen zu verbessern.

Autorenerklärung: Die Autoren erklären, dass keine finanziellen Interessenkonflikte im Zusammenhang mit diesem Beitrag bestehen.

\section{Korrespondenzadresse}

PD Dr. Sportwiss. Freerk T. Baumann

Institut für Kreislaufforschung und

Sportmedizin

Abteilung für molekulare und zelluläre

Sportmedizin

Deutsche Sporthochschule Köln

Am Sportpark Müngersdorf 6

50933 Köln

E-Mail: f.baumann@dshs-koeln.de

\section{online:}

http://dx.doi.org//10.1055/s-0035-1547546

\section{Literatur}

[1] Al-Majid S, Waters $\mathrm{H}$. The biological mechanisms of cancer-related skeletal muscle wasting: the role of progressive resistance exercise. Biol Res Nurs 2008; 10(1): 7-20

[2] Baumann FT, Zopf EM, Bloch W. Clinical exercise interventions in prostate cancer patients-a systematic review of randomized controlled trials. Support Care Cancer 2012; 20: 221-233

[3] Christensen JF, Jones LW, Tolver A, Jorgensen LW, Andersen JL, Adamsen L, Hojman P, Nielsen RH, Rorth M, Daugaard G. Safety and efficacy of resistance training in germ cell cancer patients undergoing chemotherapy: a randomized controlled trial. $\mathrm{Br}$ J Cancer 2014; 111(1): 8-16

[4] Courneya KS, Segal RJ, MackeyJR, Gelmon K, Reid RD, Friedenreich CM, Ladha AB, Proulx C, Vallance JK, Lane K, Yasui Y, McKenzie DC. Effects of aerobic and resistance exercise in breast cancer patients receiving adjuvant chemotherapy: a multicenter randomized controlled trial. J Clin Oncol 2007; 25: 43964404

[5] Kalapotharakos VI, Michalopoulou M, Godolias G, Tokmakidis SP, Malliou PV, Gourgoulis V. The effects of high- and moderateresistance training on muscle function in the elderly. J Aging Phys Act 2004; 12(2): 131 43

[6] Kenfield SA, Stampfer M], Giovannucci E, Chan JM. Physical activity and survival after prostate cancer diagnosis in the health professionals follow-up study. J Clin Oncol 2011; 29(6): 726-732 
[7] Landers J. Maximums based on reps. Natl Strength Cond Assoc J 1985; 6: 60-61

[8] Lee IM, Shiroma EJ, Lobelo F, Puska P, Blair SN, Katzmarzyk PT. Effect of physical inactivity on major non-communicable diseases worldwide: an analysis of burden of disease and life expectancy. Lancet 2012; 380 (9838): 219-29

[9] Overgård M, Angelsen A, Lydersen S, Morkved S. Does physiotherapist-guided pelvic floor muscle training reduce urinary incontinence after radical prostatectomy? A randomized controlled trial. Eur Urol 2008; 54(2): 438-48

[10] Rogers ES, MacLeod RD, Stewart ], Bird SP, Keogh JW. A randomised feasibility study of EPA and Cox-2 inhibitor (Celebrex) versus EPA, Cox-2 inhibitor (Celebrex), resistance training followed by ingestion of essential amino acids high in leucine in NSCLC cachectic patients-ACCeRT study. BMC Cancer 2011; 11: 493

[11] Scharhag-Rosenberger F, Becker T, Streckmann F, Schmidt K, Berling A, Bernardi A, Engeroff T, Exner A, Gutekunst K, Hofmeister D, Jensen W, Kähnert H, Kneis S, Limbach M, Mau-Möller A, Röcker K, Schmidt M, Schmidt T, Stöckel T, Wehrle A, Wiskemann J, Zimmer P, Zopf E, Steindorf K. Studien Zu körperlichem Training bei onkologischen Patienten: Empfehlungen zu den Erhebungsmethoden. Dtsch Z Sportmed 2014; 65(11): 304-313

[12] Schmid D, Leitzmann MF. Association between physical activity and mortality among breast cancer and colorectal cancer survivors: a systematic review and meta-analysis. Ann Oncol 2014; 25(7): 1293-1311

[13] Schmitz KH, Courneya KS, Matthews C, Demark-Wahnefried W, Galvão DA, Pinto BM, Irwin ML, Wolin KY, Segal RJ, Lucia A, Schneider CM, von Gruenigen VE, Schwartz AL. American College of Sports Medicine roundtable on exercise guidelines for cancer survivors. Med Sci Sports Exerc 2010; 42: 1409-1426

\section{Summary}

Exercise therapy is an integral part of today's oncological rehabilitation. In order to optimize the outcome of exercise programs in consideration of side effects of cancer and cancer therapies, further studies are necessary. Along with aerobic and coordination exercise, resistance training is an essential part of exercise therapy. Resistance training aids to combat side effects of lymphedema, antiandrogen therapy and cachexia among others. The present study investigated whether high load resistance training is superior to moderate resistance training regarding the increase of physical strength. For this purpose, 31 tumor patients without prior resistance training experience (different entities and therapeutic status) were randomized into either a moderate training group $(n=17)$ or a hypertrophy training group $(n=14)$. For the first 8 weeks, all patients trained in a muscular endurance circuit ( 2 rounds) consisting of 6 machines (20 repetitions on each), covering the big muscle groups. For the following 8 weeks the MT continued to lift at 20 repetitions, whereas the resistance for the HT was increased to a point where only 8-12 repetitions were possible. The maximum force was determined prior to the intervention (t0), after 8 weeks ( $\mathrm{t} 1$ ), and after 16 weeks ( $\mathrm{t} 2$ ), using a hypothetical one repetition maximum (h1RM) test. While both groups had comparable baseline levels, the results indicate that there was a significant increase in strength from $\mathrm{t} 1$ to $\mathrm{t} 2$ in the HT in almost all muscle groups. It can be concluded that a HT is vastly superior to MT regarding the increase of strength. When contraindications are absent, high load resistance training can be recommended, especially for increasing muscle mass. Whether solely the increase of strength plays a central or a peripheral role for the treatment of side effects, such as the fatigue syndrome, needs to be investigated by further studies.

Keywords: sports, exercise therapy, resistance training, cancer, supportive therapy

[14] Steindorf K, Schmidt ME, Klassen O, Ulrich CM, Oelmann J, Habermann N, Beckhove P, Owen R, Debus J, Wiskemann J, Potthoff K. Randomized controlled trial of resistance training in breast cancer patients receiving adjuvant radiotherapy: Results on cancerrelated fatigue and quality of life. Ann Oncol 2014; 11: 2237-43

[15] Stene GB, Helbostad JL, Balstad TR, Riphagen II, Kaasa S, Oldervoll LM. Effect of physical exercise on muscle mass and strength in cancer patients during treatment-a systematic review. Crit Rev Oncol Hematol 2013; 88(3): 573-93
[16] Taylor JD, Fletcher JP. Reliability of the 8-repetition maximum test in men and women. J Sci Med Sport 2014; 15: 69-73

[17] Tomlinson D, Diorio C, Beyene J, Sung L. Effect of Exercise on Cancer-Related Fatigue: A Meta-analysis. Am J Phys Med Rehabil 2014; 93: 675-686

[18] Winters-Stone KM, Dobek JC, Bennett JA, Dieckmann NF, Maddalozzo GF, Ryan CW, Beer TM. Resistance training reduces disability in prostate cancer survivors on androgen deprivation therapy: evidence from a randomized controlled trial. Arch Phys Med Rehabil 2015; 96(1): 7-14 